\title{
Hybrid Simulation in the Enterprise and Model-Based Systems Engineering
}

\section{Luis Rabelo*}

Department of Industrial Engineering \& Management, University of Central Florida, USA

Hybrid discrete-continuous simulation models of the enterprise and of the life cycle of systems are very important. This set of models consists of system dynamics models, continuous models of the higher hierarchical and more abstracted levels and connected to it are a number of discrete event simulations for selected operational, tactical functions, and phases of the life cycle. For example, system dynamics modeling best fits the macroscopic nature of activities at the higher management levels while the discrete models best fit the microscopic nature of the operational and tactical levels in the enterprise. On the other hand, system dynamics model can represent the nature of systems architecture development and discrete models the operations of a particular system.

There are several questions to be answered in the future:

- Why practitioners are not trying to develop/use hybrid models of enterprises?

- Why system engineers are not trying to integrate models of the different phases of the life cycle of a system?

- Why system engineers are not archiving in an executable way simulation models of systems of phases before op-erations has been started?

\section{Hybrid Simulation Modeling in Enterprise Management}

The management of the Enterprise is one of the classical business problems. Even components of the enterprise such as its supply chain has already enough complexity. A supply chain is a network of facilities that procure raw mate-rials/services/technologies, transform them into intermediate goods and final products/services/technologies, and deliver the products/services/technologies to customers through a distribution system. The purpose of supply chain management is to provide the right quantity of the right product at the right time to the right customers at an optimal cost. A typical manufacturing supply chain is comprised of five elements: suppliers, manufacturers, distributors, retailers and customers. The process of integrating all these elements involves the coordination and cross-functioning of production planning, purchasing, material management, production, distribution, transportation, customer service, sales forecasting, and new product/service development.

The advances in information and computing technologies in addition to the unprecedented levels of competition and fast pace of changes in the business environment have created a more flattened enterprise system and changed the way enter-prises and their supply chains should be managed. This is creating challenges to using simulation tools. For example, the presence of manufacturing and nonmanufacturing functions in the supply chain of the manufacturing enterprise, the different types of behavior in the system, differences between management levels in the scope of planning, frequency of decision making, and levels of details they deal with, indicate that a single simulation approach cannot offer all that is needed in a simulation of enterprises (Figure 1).

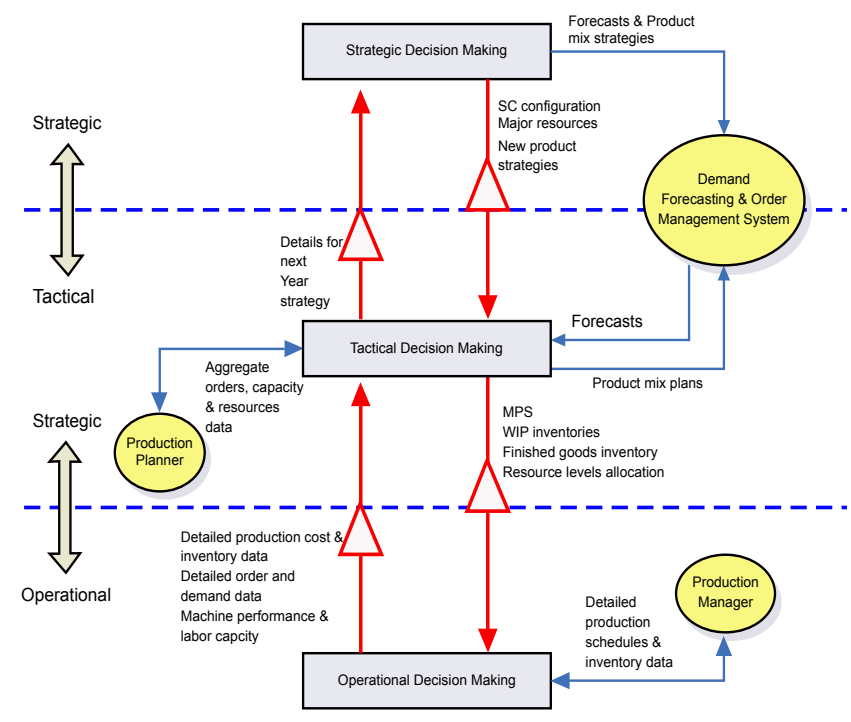

Figure 1: The manufacturing enterprise system.

\section{Hybrid Simulation Modeling in Model-Based Systems Engineering (MSBE)}

The definition of Systems Engineering used is that which is given by the International Council on Systems Engineering (INCOSE) [1] "Systems Engineering is an interdisciplinary approach and means to enable the realization of successful sys-tems. It focuses on defining customer needs and required functionality early in the development cycle, documenting requirements, and then proceeding with design synthesis and system validation while considering the complete problem". On the other hand, INCOSE [2] defines MBSE as "the formalized application of modeling to support system requirements, design, analysis, verification, and validation activities beginning in the conceptual design phase and continuing throughout development and later life cycle phases".

In the last decade, system engineers have started to replace the traditional documenting process of system engineering which has proven inappropriate for modeling the various levels of functionality

*Corresponding author: Luis Rabelo, Associate Professor, Department of Industrial Engineering \& Management, University of Central Florida, USA, Tel: (407) 882-0091; E-mail: Luis.Rabelo@ucf.edu

Received July 18, 2012; Accepted July 19, 2012; Published July 23, 2012

Citation: Rabelo L (2012) Hybrid Simulation in the Enterprise and Model-Based

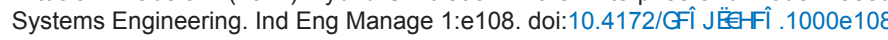

Copyright: (C) 2012 Rabelo L. This is an open-access article distributed under the terms of the Creative Commons Attribution License, which permits unrestricted use, distribution, and reproduction in any medium, provided the original author and source are credited. 


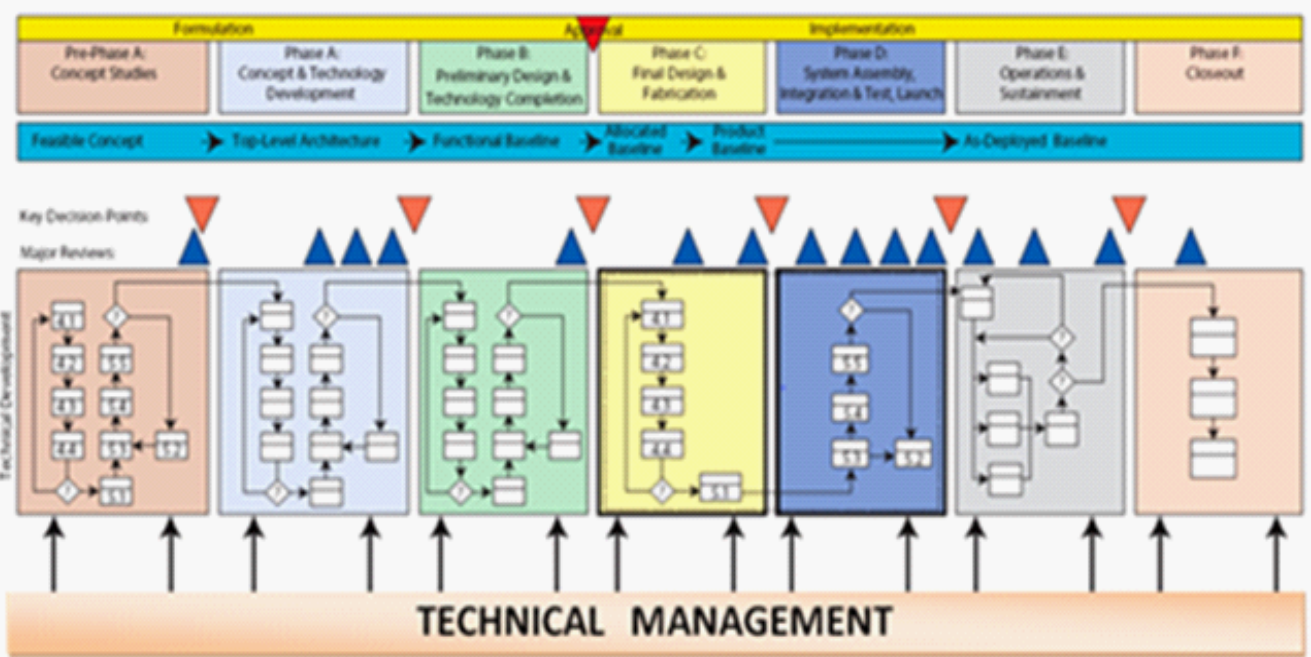

Figure 2: Life cycle according to NASA - modified from (NASA 2010).

with more simulation modeling approaches. Simulation models provide the system engineers more rigorous means for capturing and integrating system requirements, design, analysis, and verification information. However, each of these phases has required different simulation modeling artifacts (beyond the annotation type of models (Unified Modeling Language (UML)). MBSE is becoming more prevalent in the field of systems engineering. Unfortunately, the simulation models generated during the different phases of a system are from different natures and purposes. However, they are required in order to see the complete evolution of the system (even in time of future malfunctions or emergent behavior engineers cannot find the models used to design the system and cannot execute them in order to predict their behavior or they have not been updated). Hybrid simulation modeling can contribute to integrate these models in a logical and functional way (Figure 2).

\section{Conclusions}

Consequently, we have argued [3,4] that hybrid simulation modeling can contribute to offer tools/mechanisms to meet the needs created by enterprise management. In addition, we believe that hybrid simulation can also contribute to model-based systems engineering. Hybrid simulation modeling can be integrated with descriptive environments such as SysML [2] and embedded from single computer processing systems to distributed architectures using the High-Level Architecture (HLA) and the Semantic Web [5], and cloud computing. Distributed architectures can provide the recommended loosely coupled environment and standards such as HLA and the Semantic Web can facilitate interoperability. The SysML development can serve as the focal point to integrate the different simulation models.

Hybrid simulation modeling has the potential to allow industrial and system engineers to gain more effectiveness by sup-porting the modeling and analysis of complex systems pervasive to enterprise management and systems engineering [6]. In addition, developments from cloud computing, HLA, and the Semantic Web can be used to implement efficient hybrid simulation environments.

\section{References}

1. What is Systems Engineering? INCOSE International Council on Systems Engineering.
2. (2007) Systems Engineering Vision 2020, Version 2.03, TP-2004-004-02. INCOSE International Council on Systems Engineering.

3. Rabelo L, Helal M, Jones A, Min HS (2005) Enterprise simulation: A hybrid system approach. International Journal of Computer Integrated Manufacturing 18: 498-508.

4. Rabelo L, Eskandari H, Shaalan T, Helal M (2007) Value chain analysis using hybrid simulation and AHP. International Journal of Production Economics 105 536-547.

5. Tolk A, Turnitsa CD, Diallo SY, Winters LS (2006) Composable M\&S Web Services for Net-Centric Applications. Journal Defense Modeling Simulation: Applications, Methodology, Technology 3: 27-44.

6. NASA (2010) NASA Systems Engineering Handbook. 